\title{
Structural Features of Sequential Weak Measurements
}

\author{
Lajos Diós:* \\ Wigner Research Centre for Physics \\ H-1525 Budapest 114, POB 49, Hungary
}

(Dated: May 10, 2018)

\begin{abstract}
We discuss the abstract structure of sequential weak measurement (WM) of general observables. In all orders, the sequential WM correlations without post-selection yield the corresponding correlations of the Wigner function, offering direct quantum tomography through the moments of the canonical variables. Correlations in spin- $\frac{1}{2}$ sequential weak measurements coincide with those in strong measurements, they are constrained kinematically, are equivalent with single measurements. In sequential WMs with post-selection, a new anomaly occurs, different from the weak value anomaly of single WMs. In particular, the spread of polarization $\hat{\sigma}$, as measured in double WM of $\hat{\sigma}$, will diverge for certain orthogonal pre- and post-selected states.

PACS numbers: 03.65.Ta, 03.65.Wj
\end{abstract}

From textbooks on quantum mechanics we learn that the ideal measurement of observable $\hat{A}$ collapses the premeasurement state $\hat{\rho}$ into an eigenstate of $\hat{A}$ hence erasing all memory of $\hat{\rho}$. If the measurement is non-ideal (i.e.: unsharp, imprecise) the collapse still happens although it may keep some well-defined features of $\hat{\rho}$. On one hand, the larger the unsharpness the more faithfully the pre-measurement state will be preserved. On the other hand, the imprecision of the measurement can be compensated by measuring on a larger ensemble of identically prepared pre-measurement states. The concept of weak measurement (WM) corresponds to the asymptotic limit of zero precision and infinite statistics [1] when the pre-measurement state $\hat{\rho}$ would invariably survive the measurement. WM was used by Aharonov, Albert and Vaidman [2] as a non-invasive quantum measurement between pre-selection (preparation) and post-selection of the pre- and post-measurement states, respectively. Noninvasiveness of WM is a remarkable feature both with and without post-selection, and this non-invasiveness can be maintained for a succession of WMs on a single quantum system. General features of such sequential WMs form the subject of the present work.

WMs without post-selection - We outline WM of a single observable $\hat{A}$ at the abstract level of generalized (unsharp, imprecise) measurements [3]. Consider the pre-measurement state $\hat{\rho}$ and the unsharp measurement of $\hat{A}$, with precision $a$. Let $G_{a}(A)$ stand for a Gaussian function of standard width $a$. The unnormalised postmeasurement state conditioned on the outcome $A$ takes this form:

$$
\hat{\rho}_{a}(A)=\sqrt{G_{a}(A-\hat{A})} \hat{\rho} \sqrt{G_{a}(A-\hat{A})}
$$

where the outcome probability satisfies

$$
p_{a}(A)=\operatorname{tr} \hat{\rho}_{a}(A)=\left\langle G_{a}(A-\hat{A})\right\rangle_{\hat{\rho}} .
$$

If we calculate the stochastic mean $\mathbf{M} A$ of $A$ we get

$$
\mathbf{M} A=\int p_{a}(A) A \mathrm{~d} A=\langle\hat{A}\rangle_{\hat{\rho}} .
$$

We are interested in the WM limit of infinite imprecision $a \rightarrow \infty$, i.e., when $a$ is so large that the difference between pre- and post-measurement states is negligible. In practice it means $a \gg \Delta A$ where $(\Delta A)^{2}=\left\langle\hat{A}^{2}\right\rangle_{\hat{\rho}}-\left(\langle\hat{A}\rangle_{\hat{\rho}}\right)^{2}$. While the relationship $\mathbf{M} A$ is independent of $a$ the probability distribution $p_{a}(A)$ diverges so that $p_{\infty}(A)$ does not exist. Note with ref. [1] the WM limit of the unsharp measurement (12) had been used earlier for theory of time-continuous measurement [4].

Before constructing sequential WMs, let us write the post-measurement state (1) into the equivalent form:

$$
\hat{\rho}_{a}(A)=\exp \left(\frac{-\hat{A}_{\Delta}^{2}}{8 a^{2}}\right) G_{a}\left(A-\hat{A}_{c}\right) \hat{\rho}
$$

where $\hat{A}_{\Delta}, \hat{A}_{c}$ are commuting superoperators [5] defined by $\hat{A}_{\Delta} \hat{O}=[\hat{A}, \hat{O}]$ and $\hat{A}_{c} \hat{O}=\frac{1}{2}\{\hat{A}, \hat{O}\}$. As an example of sequential WMs, we consider the sequence of three independent WMs of $\hat{A}, \hat{B}, \hat{C}$, in this order. In the WM limit, we can apply eq. (4) without the exponential factor to construct the unnormalized post-measurement state:

$$
\hat{\rho}_{a}(A, B, C)=G_{a}\left(C-\hat{C}_{c}\right) G_{a}\left(B-\hat{B}_{c}\right) G_{a}\left(A-\hat{A}_{c}\right) \hat{\rho} .
$$

The joint probability distribution of the three outcomes is determined by the trace of the post-measurement state:

$$
\begin{aligned}
& p_{a}(A, B, C)=\operatorname{tr} \hat{\rho}_{a}(A, B, C)= \\
= & \operatorname{tr}\left\{G_{a}\left(C-\hat{C}_{c}\right) G_{a}\left(B-\hat{B}_{c}\right) G_{a}\left(A-\hat{A}_{c}\right) \hat{\rho}\right\},
\end{aligned}
$$

which, as we said already, diverges in the WM limit and $p_{\infty}(A, B, C)$ does not exist. Nonetheless, the stochastic average of the product $\mathrm{ABC}$ is independent of $a$ in the WM limit. Using eq. (6), we obtain

$$
\begin{aligned}
\mathbf{M} A B C & =\int p_{a}(A, B, C) A B C \mathrm{~d} A \mathrm{~d} B \mathrm{~d} C= \\
& =\frac{1}{8}\langle\{\hat{A},\{\hat{B}, \hat{C}\}\}\rangle_{\hat{\rho}} .
\end{aligned}
$$

This important result was obtained by Bednorz and Belzig [6] assuming a quasi-distribution which this time we justify as follows. 
Since the r.h.s. of the above expression is independent of $a$ therefore we can calculate it for $a=0$. This means, we get the following quasi-distribution from the true $p_{a}(A, B, C)$ :

$$
p_{0}(A, B, C)=\operatorname{tr}\left\{\delta\left(C-\hat{C}_{c}\right) \delta\left(B-\hat{B}_{c}\right) \delta\left(A-\hat{A}_{c}\right) \hat{\rho}\right\} .
$$

This quasi-distribution can have negative domains. (For the true distribution $p_{a}(A, B, C) \geq 0$ holds in the WM limit.) The merit of this quasi-distribution is that it does not contain the diverging parameter $a$ and yields correctly the mean for the product $A B C$ exactly like $p_{a}(A, B, C)$ did:

$$
\begin{aligned}
\mathbf{M} A B C & =\frac{1}{8}\langle\{\hat{A},\{\hat{B}, \hat{C}\}\}\rangle_{\hat{\rho}}= \\
& =\int p_{0}(A, B, C) A B C \mathrm{~d} A \mathrm{~d} B \mathrm{~d} C .
\end{aligned}
$$

The same is true for the means of $A, B, C, A B, A C, B C$, respectively. But all other means diverge in reality, i.e.: with $p_{a}(A, B, C)$ in the WM limit, whereas $p_{0}(A B C)$ suggests incorrect finite values for them.

The above results can trivially be extended for an arbitrary long sequence of WMs. Let us consider a sequence of observables $\hat{A}_{1}, \hat{A}_{2}, \ldots, \hat{A}_{n}$ which are weakly measured in the given order on the pre-measurement state $\hat{\rho}$. If $A_{1}, A_{2}, \ldots, A_{n}$ denote the corresponding measurement outcomes then

$$
\mathbf{M} A_{1} A_{2} \ldots A_{n}=\frac{1}{2^{n}}\left\langle\left\{\hat{A}_{1},\left\{\hat{A}_{2},\left\{\ldots,\left\{\hat{A}_{n-1}, \hat{A}_{n}\right\} \ldots\right\}\right\}\right\}\right\rangle_{\hat{\rho}} .
$$

The stochastic mean of the product of sequential WM outcomes coincides with the quantum expectation value of the stepwise-symmetrized (also called time-symmetric [7) product of quantum observables. This is the central result for sequential WMs without post-selection. If we regard a subset of the $n$ outcomes and discard the rest of them then the similar identity holds, e.g.: $\mathbf{M} A_{2} A_{7} A_{8}=$ $\frac{1}{8}\left\langle\left\{\hat{A}_{2},\left\{\hat{A}_{7}, \hat{A}_{8}\right\}\right\}\right\rangle_{\hat{\rho}}$. In general,

$$
\mathbf{M} A_{i_{1}} \ldots A_{i_{2}} \ldots A_{i_{r}}=\frac{1}{2^{r}}\left\langle\left\{\hat{A}_{i_{1}},\left\{\hat{A}_{i_{2}}, \ldots, \hat{A}_{i_{r}}\right\}\right\}\right\rangle_{\hat{\rho}}
$$

holds for $\left(i_{1}, i_{2}, \ldots, i_{r}\right) \subseteq(1,2, \ldots, n)$, i.e., for any ordered subsets of indices from 1 to $n$, as it follows easily from the derivation of eq. (10). Also we can derive all the above stochastic means from the quasi-distribution:

$$
\begin{aligned}
& p_{0}\left(A_{1}, A_{2}, \ldots, A_{n}\right)= \\
= & \operatorname{tr}\left\{\delta\left(A_{n}-\hat{A}_{1, c}\right) \delta\left(A_{2}-\hat{A}_{2, c}\right) \ldots \delta\left(A_{n}-\hat{A}_{n, c}\right) \hat{\rho}\right\} .
\end{aligned}
$$

Generalization of the relationship (91) holds:

$$
\begin{gathered}
\mathbf{M} A_{1} A_{2} \ldots A_{n}=\frac{1}{2^{n}}\left\langle\left\{\hat{A}_{1},\left\{\ldots,\left\{\hat{A}_{n-1}, \hat{A}_{n}\right\} \ldots\right\}\right\}\right\rangle_{\hat{\rho}}= \\
=\int p_{0}\left(A_{1}, A_{2}, \ldots, A_{n}\right) A_{1} A_{2} \ldots A_{n} \mathrm{~d} A_{1} \mathrm{~d} A_{2} \ldots \mathrm{d} A_{n} .(13)
\end{gathered}
$$

The last two WMs in a stepwise-symmetrized sequence are always interchangeable but the rest of them are not: order of WMs matters in general. There is, however, a remarkable class when all WMs are interchangeable. Let us discuss the example of the sequence $\hat{A}, \hat{B}, \hat{C}$. To find a simplest sufficient condition of complete interchangeability, we require that the superoperators $\hat{A}_{c}, \hat{B}_{c}, \hat{C}_{c}$ in eq. (77) all commute. Take, e.g., the identity $\left[\hat{A}_{c}, \hat{B}_{c}\right] \hat{O}=$ $\frac{1}{4}[[\hat{A}, \hat{B}], \hat{O}]$ which says that $\hat{A}_{c}$ and $\hat{B}_{c}$ commute if $[\hat{A}, \hat{B}]$ is a c-number. Therefore the interchangeability of the three WMs is ensured if all three commutators $[\hat{A}, \hat{B}]$, $[\hat{A}, \hat{C}]$ and $[\hat{B}, \hat{C}]$ are c-numbers. In the general case, the order of WMs within the sequence $\hat{A}_{1}, \hat{A}_{2}, \ldots, \hat{A}_{n}$ becomes irrelevant if

$$
\left[\hat{A}_{k}, \hat{A}_{l}\right]=\text { c-number } \quad(k, l=1,2, \ldots, n) .
$$

This is not necessary just a sufficient condition of complete interchangeability of the $n$ WMs. Under this condition, the stepwise-symmetrization on the r.h.s. of eq. (10) reduces to symmetrization:

$$
\mathbf{M} A_{1} A_{2} \ldots A_{n}=\left\langle\mathcal{S} \hat{A}_{1} \hat{A}_{2} \ldots \hat{A}_{n}\right\rangle_{\hat{\rho}},
$$

where $\mathcal{S}$ stands for symmetrization of the operator product behind it.

Canonical observables - The conditions (14) hold typically for the linear combination of canonical variables, e.g., for the choice

$$
\hat{A}_{k}=u_{k} \hat{q}+v_{k} \hat{p} \quad(k=1,2, \ldots, n),
$$

where $[\hat{q}, \hat{p}]=i$. Then symmetrization $\mathcal{S}$ is nothing else than Weyl ordering. Since the Weyl-ordered correlation functions of canonical variables $\hat{q}, \hat{p}$, or of their linear combinations like on r.h.s. of eq. (15) coincide with the corresponding correlation functions (moments) calculated from the Wigner function $W(q, p)$ of $\hat{\rho}$, we conclude that the r.h.s. can be re-written in terms of Wigner function correlations:

$$
\begin{aligned}
\mathbf{M} A_{1} A_{2} \ldots A_{n} & =\int W(q, p) A_{1} A_{2} \ldots A_{n} \mathrm{~d} q \mathrm{~d} p \equiv \\
& \equiv\left\langle A_{1} A_{2} \ldots A_{n}\right\rangle_{W} .
\end{aligned}
$$

This means that for sequential WMs of canonical observables the generic quasi-distribution (12) is redundant for $n>2$, its role is taken over by the Wigner quasidistribution. The coincidence $p_{0}(q, p)=W(q, p)$ in the special case $n=2, \hat{A}_{1}=\hat{q}, \hat{A}_{2}=\hat{p}$ was recognized in [6]

Suppose, for instance, we perform two WMs of $\hat{q}$ with outcomes $q_{1}, q_{2}$ and two WMs of $\hat{p}$ with outcomes $p_{1}, p_{2}$. Then independently of the orders of the four WMs, a sufficiently large statistics of outcomes allows us to determine all second order moments of the Wigner function

$$
\begin{aligned}
\left\langle q^{2}\right\rangle_{W} & =\mathbf{M} q_{1} q_{2}, \quad\left\langle p^{2}\right\rangle_{W}=\mathbf{M} p_{1} p_{2}, \\
\langle q p\rangle_{W} & =\mathbf{M} q_{1} p_{1}=\mathbf{M} q_{1} p_{2}=\mathbf{M} q_{2} p_{1}=\mathbf{M} q_{2} p_{2}
\end{aligned}
$$


as well as a few higher order ones $\left\langle q^{2} p\right\rangle_{W},\left\langle q p^{2}\right\rangle_{W}$, $\left\langle q^{2} p^{2}\right\rangle_{W}$ and, of course, the first order moments $\langle q\rangle_{W}$, $\langle p\rangle_{W}$, too.

Spin- $\frac{1}{2}$ observables - Sequential measurement of spin$\frac{1}{2}$ observables is exceptional: eq. (10) is valid no matter the measurements are weak, ideal (strong), or even alternating within the sequence between the two extreme strength. Consider the following choice of observables:

$$
\hat{A}_{1}=\hat{\sigma}_{1}, \hat{A}_{2}=\hat{\sigma}_{2}, \ldots, \hat{A}_{n}=\hat{\sigma}_{n}
$$

where $\hat{\sigma}_{k}$ is the polarization parallel to the unit vector $\vec{e}_{k}$ for $k=1,2, \ldots, n$. Denote the measurement outcomes by $A_{1}=\sigma_{1}, A_{2}=\sigma_{2}$, etc., and invoke eq. (10) form them:

$$
\mathbf{M} \sigma_{1} \sigma_{2} \ldots \sigma_{n}=\frac{1}{2^{n}}\left\langle\left\{\hat{\sigma}_{1},\left\{\hat{\sigma}_{2},\left\{\ldots,\left\{\hat{\sigma}_{n-1}, \hat{\sigma}_{n}\right\} \ldots\right\}\right\}\right\}\right\rangle_{\hat{\rho}}
$$

To confirm it for strong measurements as well, we introduce the projectors $\hat{P}_{ \pm}=\frac{1}{2}(1 \pm \hat{\sigma})$ diagonalizing the Pauli polarization matrix $\hat{\sigma}$. Standard expression for sequential strong measurements reads:

$$
\mathbf{M} \sigma_{1} \sigma_{2} \ldots \sigma_{n}=\operatorname{tr} \sum_{\sigma_{n}= \pm 1} \sigma_{n} \hat{P}_{\sigma_{n}}^{(n)} \ldots\left(\sum_{\sigma_{2}= \pm 1} \sigma_{2} \hat{P}_{\sigma_{2}}^{(2)}\left(\sum_{\sigma_{1}= \pm 1} \sigma_{1} \hat{P}_{\sigma_{1}}^{(1)} \hat{\rho} \hat{P}_{\sigma_{1}}^{(1)}\right) \hat{P}_{\sigma_{2}}^{(2)}\right) \ldots \hat{P}_{\sigma_{n}}^{(n)}
$$

Observe the identity $\sum_{\sigma= \pm} \sigma \hat{P}_{a} \hat{O} \hat{P}_{a}=\frac{1}{2}\{\hat{\sigma}, \hat{O}\}$ valid for auxiliary $2 \times 2$ matrices $\hat{O}$, apply it $n$-times. We obtain eq. (20). Evaluating its r.h.s. yields

$$
\mathbf{M} \sigma_{1} \sigma_{2} \ldots \sigma_{n}=\left\{\begin{array}{cc}
\left(\vec{e}_{1} \vec{e}_{2}\right)\left(\vec{e}_{3} \vec{e}_{4}\right) \ldots\left(\vec{e}_{n-1} \vec{e}_{n}\right) & n \text { even } \\
\left\langle\hat{\sigma}_{1}\right\rangle_{\hat{\rho}}\left(\vec{e}_{2} \vec{e}_{3}\right) \ldots\left(\vec{e}_{n-1} \vec{e}_{n}\right) & n \text { odd }
\end{array}\right.
$$

Outcome correlations of $n$ sequential WMs on a spin$\frac{1}{2}$ system coincide exactly with the correlations obtained from strong measurements of the same sequence. Correlations are kinematically constrained by the chosen directions of polarization measurements. For $n$ even, correlations are completely determined by the single mean $\left\langle\hat{\sigma}_{1}\right\rangle_{\hat{\rho}}$ and just independent of the pre-measurement state $\hat{\rho}$ if $n$ is even.

WMs with post-selection - So far we have established the general features of outcome statistics in sequential WMs without post-selection. Including post-selection requires straightforward modifications. For mixed state post-selection [1, 8], the statistics (6) of the $A B C$ sequential WM modifies like this:

$$
\begin{aligned}
& p_{a}(A, B, C \mid \hat{\Pi})=\frac{\operatorname{tr}\left\{\hat{\Pi} \hat{\rho}_{a}(A, B, C)\right\}}{\operatorname{tr}\{\hat{\Pi} \hat{\rho}\}}= \\
= & \frac{\operatorname{tr}\left\{\hat{\Pi} G_{a}\left(C-\hat{C}_{c}\right) G_{a}\left(B-\hat{B}_{c}\right) G_{a}\left(A-\hat{A}_{c}\right) \hat{\rho}\right\}}{\operatorname{tr}\{\hat{\Pi} \hat{\rho}\}}
\end{aligned}
$$

where $0 \leq \hat{\Pi} \leq 1$. Accordingly, the post-selected mean (77), i.e., the mean restricted for the post-selected subset $\left.A B C\right|_{p s e l}$ of $A B C$, becomes

$$
\left.\mathbf{M} A B C\right|_{p s e l}=\frac{1}{8}\langle\{\hat{A},\{\hat{B},\{\hat{C}, \hat{\Pi}\}\}\}\rangle_{\hat{\rho}} /\langle\hat{\Pi}\rangle_{\hat{\rho}} .
$$

The general result must be the following:

$$
\mathbf{M} A_{1}, A_{2}, \ldots,\left.A_{n}\right|_{p s e l}=\frac{\left\langle\left\{\hat{A}_{1},\left\{\hat{A}_{2}, \ldots,\left\{\hat{A}_{n}, \hat{\Pi}\right\} \ldots\right\}\right\}\right\rangle_{\hat{\rho}}}{2^{n}\langle\hat{\Pi}\rangle_{\hat{\rho}}} .
$$

In the basic case, both initial and post-selected states are pure states and we are going to take this option: $\hat{\rho}=|i\rangle\langle i|, \hat{\Pi}=| f\rangle\langle f|$. Then, following Mitchison, Jozsa and Popescu, we introduce the sequential weak values

$$
\left(A_{1}, A_{2}, \ldots, A_{n}\right)_{w}=\frac{\left\langle f\left|\hat{A}_{n} \hat{A}_{n-1} \ldots \hat{A}_{1}\right| i\right\rangle}{\langle f \mid i\rangle},
$$

and re-write eq. (24) in time-symmetric form [9]:

$$
\begin{gathered}
\mathbf{M} A_{1}, A_{2}, \ldots,\left.A_{n}\right|_{p s e l}= \\
=\frac{1}{2^{n}} \sum\left(A_{i_{1}}, A_{i_{2}}, \ldots, A_{i_{r}}\right)_{w}\left(A_{j_{1}}, A_{j_{2}}, \ldots, A_{j_{n-r}}\right)_{w}^{\star}
\end{gathered}
$$

where summation is understood for all partitions $\left(i_{1}, i_{2}, \ldots, i_{r}\right) \cup\left(j_{1}, j_{2}, \ldots, j_{n-r}\right)=(1,2, \ldots, n)$ where $i$ 's and $j$ 's remain ordered. Degenerate partitions $r=0, n$, too, must be counted. Certain options of reduction, shown above for sequential WMs of canonical or spin$\frac{1}{2}$ observables, may still survive post-selection, here we are not going to discuss them. We show a particular anomaly, not present in single post-selected WM but in sequential WMs, even for simplest ones.

Re-selection - Consider the special case $|i\rangle=|f\rangle$ of post-selection, call it re-selection. In the case of a single $\mathrm{WM}$, re-selection is equivalent with no post-selection:

$$
\mathbf{M} A=\left.\mathbf{M} A\right|_{r s e l}=\langle\hat{A}\rangle_{\hat{\rho}} .
$$

Since WMs are considered non-invasive, we expect that the post-measurement state does not differ from the initial state $|i\rangle$ in the WM limit, re-selection rate tends to 1 
hence the discarded outcomes would not alter the statistics. No doubt, this is the case for a single WM. As to sequential WMs, however, a glance at (26) shows that reselection does not yield equivalent results with no postselection (10). Even the simplest sequential WM will illustrate the anomaly. We consider two WMs, moreover, we consider the case when $\hat{A}_{1}=\hat{A}_{2}=\hat{A}$, i.e, we weakly measure $\hat{A}$ twice in a row, yielding outcomes $A_{1}$ and $A_{2}$, respectively. Without post-selection, eq. (10) and with re-selection eq. (26) yield, respectively:

$$
\begin{aligned}
\mathbf{M} A_{1} A_{2} & =\left\langle i\left|\hat{A}^{2}\right| i\right\rangle, \\
\left.\mathbf{M} A_{1} A_{2}\right|_{\text {rsel }} & =\frac{1}{2}\left\langle i\left|\hat{A}^{2}\right| i\right\rangle+\frac{1}{2}(\langle i|\hat{A}| i\rangle)^{2} .
\end{aligned}
$$

Re-selection decreases $\mathbf{M} A_{1} A_{2}$ by half of the squared quantum uncertainty $(\Delta A)^{2}$ in state $|i\rangle$ :

$$
\mathbf{M} A_{1} A_{2}-\left.\mathbf{M} A_{1} A_{2}\right|_{r s e l}=\frac{1}{2}(\Delta A)^{2} .
$$

This is an unexpected anomaly. The reason must lie in the contribution of outcomes discarded by re-selection, i.e.: $\left.\mathbf{M} A_{1} A_{2}\right|_{\text {disc }} \times($ discard rate $) \rightarrow \frac{1}{2}(\Delta A)^{2}$ must be satisfied.

As an example, consider a spin- $\frac{1}{2}$ system in upward polarized initial state $|i\rangle=|\uparrow\rangle$. Let us begin with a single WM of $\hat{\sigma} \equiv \hat{\sigma}_{x}$ with outcome $\sigma_{1}$. The contribution of the discarded outcomes reads

$$
\left.\mathbf{M} \sigma_{1}\right|_{\text {disc }}=\frac{\left\langle\downarrow\left|\left(\exp \left(-\frac{1}{8} \hat{\sigma}_{\Delta}^{2} / a^{2}\right) \hat{\sigma}_{c}|\uparrow\rangle\langle\uparrow|\right)\right| \downarrow\right\rangle}{\left\langle\downarrow\left|\left(\exp \left(-\frac{1}{8} \hat{\sigma}_{\Delta}^{2} / a^{2}\right)|\uparrow\rangle\langle\uparrow|\right)\right| \downarrow\right\rangle}
$$

where we use the exact expression of the post-WM state with the exponential factor as in eq. (44) otherwise we get 0 for the rate of discarded events. This rate is just the denominator in the above fraction, yielding $\sim \frac{1}{4} a^{-2}$ asymptotically. This rate goes to zero in the WM limit but $\left.\mathbf{M} \sigma_{1}\right|_{\text {disc }}$ vanishes anyway since the numerator is zero identically. Now, let us weakly measure $\hat{\sigma} \equiv \hat{\sigma}_{x}$ twice in a sequence, yielding outcomes $\sigma_{1}, \sigma_{2}$. Since the quantum spread $\Delta \sigma_{x}=1$ in state $|\uparrow\rangle$, we have to prove that in reselection the contribution of the discarded events satisfies $\left.\mathbf{M} \sigma_{1} \sigma_{2}\right|_{\text {disc }} \times($ discard rate $) \rightarrow \frac{1}{2}$. Its analytic form can be written as

$$
\left.\mathbf{M} \sigma_{1} \sigma_{2}\right|_{\text {disc }}=\frac{\left\langle\downarrow\left|\left(\exp \left(-\frac{1}{4} \hat{\sigma}_{\Delta}^{2} / a^{2}\right) \hat{\sigma}_{c}^{2}|\uparrow\rangle\langle\uparrow|\right)\right| \downarrow\right\rangle}{\left\langle\downarrow\left|\left(\exp \left(-\frac{1}{4} \hat{\sigma}_{\Delta}^{2} / a^{2}\right)|\uparrow\rangle\langle\uparrow|\right)\right| \downarrow\right\rangle} .
$$

The denominator yields rate $\sim \frac{1}{2} a^{-2}$ of discards, it is vanishing in the WM limit. The exponential factor in the numerator can be neglected in the WM limit and we get the following result:

$$
\begin{aligned}
\left.\mathbf{M} \sigma_{1} \sigma_{2}\right|_{\text {disc }} & =2 a^{2}\left\langle\downarrow\left|\left(\hat{\sigma}_{c}^{2}|\uparrow\rangle\langle\uparrow|\right)\right| \downarrow\right\rangle= \\
& =2 a^{2} \frac{1}{4}\langle\downarrow|\{\hat{\sigma},\{\hat{\sigma},|\uparrow\rangle\langle\uparrow|\}\}| \downarrow\rangle=a^{2} .
\end{aligned}
$$

As we see, the correlation of two subsequent $\hat{\sigma}_{x}$ polarization WMs diverges on the discarded events in reselection. This is in itself a different and stronger anomaly than the paradigmatic large but finite mean values obtained in single WMs with post-selection [2]. What we wished to confirm here is that the divergent mean $a^{2}$ compensates the vanishing rate $\frac{1}{2} a^{-2}$ to yield the finite contribution $\frac{1}{2}$ of the discarded outcomes in re-selection.

Summary, discussion - Superoperator formalism has helped us to determine the correlation functions of sequential WMs in terms of the quantum expectation values of the step-wise symmetric product of the corresponding observables. Condition of interchangeability of WMs within the sequence has been found. Canonical variables are interchangeable and, without post-selection, their WM correlation functions coincide with the corresponding correlation functions of the Wigner function. It follows from our result how all $n$-th order correlation functions (moments) of the Wigner function can, in principle, be determined directly on the outcome statistics of the sequence of $n$ WMs. This makes sequential WMs a tool of direct quantum state tomography (limited normally by the highest available order $n$ in a given experiment). Sequential WMs may demonstrate quantum paradoxes since the negativity of the Wigner function leads to non-classical statistics of sequential WMs, like in ref. [6], see also [10]. Earlier suggestions associated outcomes of single post-selected WMs with Bohmian velocities [11]. As to the outcomes of sequential WM, our result suggests Wigner phase space coordinates as the natural interpretation. (This interpretation proves to be universal if sequential WM of spin- $\frac{1}{2}$ observables is related to Wigner function in Grassmann variables introduced in ref. [12], an issue we leave open here.) Spin$\frac{1}{2}$ observables behave very differently. Two polarization WMs yield no new information at all compared to single measurements since the correlation is determined by the angle between the two polarizers and independent of the quantum state, just like for two strong (ideal) polarization measurements. This is more than resemblance. We found that a sequence of $n$ weak or, alternatively, strong spin- $\frac{1}{2}$ measurements yield identical $n$-order correlation functions, respectively.

Finally, we studied the marginal case $|f\rangle=|i\rangle$ of postselection which we called re-selection and found that in sequential WMs it is not equivalent with lack of postselection. This means that in sequential WMs with reselection the discarded statistics matters however close we are to ideal WMs. This unexpected effect roots in a novel weak value anomaly this time referring to the anomalous (divergent) value of the weakly measured (i.e.: in sequential WM) auto-correlation on the statistics discarded by re-selection. This phenomenon is a robust feature of sequential WMs and it is not tractable in terms of standard weak values. As an example, we have shown that the correlation of outcomes in double WM of $\hat{\sigma}_{x}$ in pre-selected state $|i\rangle=|\uparrow\rangle$ and post-selected on $|f\rangle=|\downarrow\rangle$ will diverge whereas any correlation larger than $\left\|\sigma_{x}\right\|^{2}=1$ is counter-intuitive. 
This work was supported by the Hungarian Scientific Research Fund under Grant No. 103917, and by the EU COST Action MP1209.

* Electronic address: diosi.lajos@wigner.mta.hu

[1] L. Diósi: Quantum mechanics: weak measurements, v4, p276 in: Encyclopedia of Mathematical Physics, eds.: J.P. Françoise, G.L. Naber, and S.T. Tsou (Elsevier, Oxford, 2006).

[2] Y. Aharonov, D.Z. Albert and L. Vaidman, Phys. Rev. Lett. 60, 1351 (1988).

[3] K. Kraus, States, Effects and Operations (Springer, Berlin, 1983); M.A. Nielsen and I.L. Chuang: Quantum Computation and Quantum Information (Cambridge University Press, Cambridge, 2000).

[4] A. Barchielli, L. Lanz and G.M. Prosperi, Nuovo Cim. 72B, 79 (1982); C.M. Caves and G.J. Milburn, Phys. Rev. A 36, 5543 (1987); L. Diósi, Phys. Lett. 129A, 419
(1988).

[5] K. Chou, Z. Su, B. Hao, and L. Yu, Phys. Rep. 118, 1 (1985); L. Diósi, Phys. Rev. A 42, 5086 (1990); Quant. Semiclass. Opt. 8, 309 (1996).

[6] A. Bednorz and W. Belzig, Phys. Rev. Lett. 105, 106803 (2010).

[7] B. Berg, L. I. Plimak, A. Polkovnikov, M. K. Olsen, M. Fleischhauer, and W. P. Schleich Phys. Rev. A 80, 033624 (2009).

[8] R. Silva, Y. Guryanova, N. Brunner, N. Linden, A. J. Short, and S. Popescu, Phys. Rev. A 89, 012121 (2014).

[9] G. Mitchison, R. Jozsa, and S. Popescu, Phys. Rev. A 76, 062105 (2007).

[10] A. Bednorz and W. Belzig, Phys. Rev. A 83, 052113 (2011).

[11] H. M. Wiseman, New J. Phys. 9, 165 (2007); S. Kocsis, B. Braverman, S. Ravets, M. J. Stevens, R. P. Mirin, L. K. Shalm, and A. M. Steinberg, Science 332, 1170 (2011).

[12] K. E. Cahill and R. J. Glauber, Phys. Rev. A 59, 1538 (1999). 